\title{
Green tea polyphenol epigallocatechin-3-gallate ameliorates insulin resistance in non-alcoholic fatty liver disease mice
}

\author{
Lu GAN ${ }^{1, \#}$, Zi-jun MENG ${ }^{1, \#}$, Ri-bo XIONG ${ }^{2, \#, ~ J i n-q i a n g ~ G U O ~}{ }^{1}$, Xiao-cui LU ${ }^{1}$, Zhi-wei ZHENG ${ }^{1}$, Yan-ping DENG ${ }^{1}$, Bing-de LUO ${ }^{1}$, \\ Fei $\mathrm{ZOU}^{3, *}$, Hua $\mathrm{LI}^{1, *}$ \\ ${ }^{1}$ Experimental Teaching Center of Preventive Medicine, Guangdong Provincial Key Laboratory of Tropical Disease Research, School \\ of Public Health and Tropical Medicine, Southern Medical University, Guangzhou 510515, China; ${ }^{2}$ Department of Rehabilitation, \\ The Third Affiliated Hospital of Southern Medical University, Guangzhou 510630, China; ${ }^{3}$ Department of Occupational Health and \\ Occupational Medicine, Guangdong Provincial Key Laboratory of Tropical Disease Research, School of Public Health and Tropical \\ Medicine, Southern Medical University, Guangzhou 510515, China
}

Aim: Epigallocatechin-3-gallate (EGCG) is a major polyphenol in green tea. In this study, we investigated the effects of EGCG on insulin resistance and insulin clearance in non-alcoholic fatty liver disease (NAFLD) mice.

Methods: Mice were fed on a high-fat diet for 24 weeks. During the last 4 weeks, the mice were injected with EGCG (10, 20 and 40 $\mathrm{mg} \cdot \mathrm{kg}^{-1} \cdot \mathrm{d}^{-1}$, ip). Glucose tolerance, insulin tolerance and insulin clearance were assessed. After the mice were euthanized, blood samples and tissue specimens were collected. Glucose-stimulated insulin secretion was examined in isolated pancreatic islets. The progression of NAFLD was evaluated histologically and by measuring lipid contents. Insulin-degrading enzyme (IDE) protein expression and enzyme activity were detected using Western blot and immunocapture activity assays, respectively.

Results: The high-fat diet significantly increased the body weight and induced grade 2 or 3 liver fatty degeneration (steatosis, lobular inflammation and ballooning) accompanied by severe hyperlipidemia, hyperglycemia, hyperinsulinemia and insulin resistance in the model mice. Administration of EGCG dose-dependently ameliorated the hepatic morphology and function, reduced the body weight, and alleviated hyperlipidemia, hyperglycemia, hyperinsulinemia and insulin resistance in NAFLD mice. Furthermore, EGCG dosedependently enhanced insulin clearance and upregulated IDE protein expression and enzyme activity in the liver of NAFLD mice. Conclusion: EGCG dose-dependently improves insulin resistance in NAFLD mice not only by reducing body weight but also through enhancing the insulin clearance by hepatic IDE. The results suggest that IDE be a potential drug target for the treatment of NAFLD.

Keywords: epigallocatechin-3-gallate; polyphenol; green tea; non-alcoholic fatty liver disease; metabolic disorders; insulin resistance; insulin clearance; insulin-degrading enzyme

Acta Pharmacologica Sinica (2015) 36: 597-605; doi: 10.1038/aps.2015.11; published online 20 Apr 2015

\section{Introduction}

Non-alcoholic fatty liver disease (NAFLD) is considered to be the most common cause of chronic liver disease worldwide and is a multifactorial disease with a complex pathophysiology, including hepatic steatosis, non-alcoholic steatohepatitis (NASH), and subsequent fibrosis, cirrhosis and hepatocellular carcinoma. Insulin resistance is closely associated with NAFLD and is a risk factor for NAFLD ${ }^{[1,2]}$. It has been shown

\footnotetext{
\# These authors contributed equally to this article.

* To whom correspondence should be addressed.

E-mail zoufei616@163.com (Fei ZOU); lih@smu.edu.cn (Hua LI)

Received 2014-10-28 Accepted 2015-02-27
}

that a chronic exposure of tissues to high insulin levels (ie, hyperinsulinemia) induces insulin resistance ${ }^{[3,4]}$. The circulating insulin level depends on a tightly regulated balance between insulin secretion and clearance ${ }^{[5]}$. Much consideration has been given to the importance of insulin secretion in the development of hyperinsulinemia and insulin resistance. However, relatively less attention has been directed to the role of insulin clearance in NAFLD, which is an important determinant of plasma insulin.

Insulin clearance is mostly controlled by the liver. Usually, $50 \%-80 \%$ of insulin is cleared upon degradation by the insulin-degrading enzyme (IDE) in the liver, which is a ratelimiting enzyme in the insulin degradation process ${ }^{[6]}$. IDE is an intracellular $110-\mathrm{kDa}$ metalloproteinase located in the 
cytosol, peroxisomes and endosomes. It catalyzes the degradation of several proteins, including insulin, amylin and $\beta$-amyloid. Several human genetic studies have demonstrated that IDE gene polymorphisms and mutations are associated with insulin resistance ${ }^{[7]}$. The inactivation of IDE by gene mutation or gene knockout induces hyperinsulinemia and insulin resistance in both rats and mice ${ }^{[8,9]}$. These findings suggest that IDE is important in maintaining normal plasma insulin levels and insulin sensitivity. A recent study has shown a close relationship between disease severity, hyperinsulinemia and impaired insulin clearance in NASH patients ${ }^{[10]}$. However, this study did not mention whether IDE is involved in NAFLD, which is an important enzyme mediating insulin clearance.

Epigallocatechin-3-gallate (EGCG) is the most abundant catechin extracted from natural green tea. EGCG has been extensively studied for its beneficial effects on cardiovascular diseases, cancer and inflammation, has negligible side effects and is relatively inexpensive compared to conventional pharmaceutical drugs ${ }^{[11]}$. Recent studies have shown a strong correlation between the consumption of green tea and the prevention of NAFLD. Other studies have shown that EGCG reduced the severity of liver injury by reducing circulating insulin, improving insulin sensitivity and attenuating insulin resistance in type 2 diabetes mellitus (T2DM) or NAFLD mice $^{[12-14]}$. Although EGCG reduces circulating insulin, it is not clear whether IDE is involved. Any effects of EGCG on insulin clearance by hepatic IDE in NAFLD have not been reported. In this study, we investigated the effects of EGCG on insulin clearance and insulin resistance in NAFLD mice induced by a high-fat diet, and evaluated the effects of EGCG on the activity and expression of hepatic IDE.

\section{Materials and methods}

\section{Green tea extracted EGCG}

High purity EGCG (99\%) extracted from green tea was provided by Gosun Biotechnologies Co, Ltd (Hangzhou, China). EGCG was freshly prepared in $0.9 \% \mathrm{NaCl}$ solution before use.

\section{Animal experimental procedures}

All experiments were performed according to the National Institute of Health Guide for the Care and Use of Laboratory Animals and were approved by the Experimental Animals Ethics Committee of Nanfang Medical University. Male C57BL/6 mice that were 6-8 weeks old were acquired from the Guangdong Medical Laboratory Animal Center and were housed in animal quarters at $22^{\circ} \mathrm{C}$ with a $12 \mathrm{~h}$ light-dark cycle, and after 1 week in quarantine, the mice were randomly divided into two groups: a control group $(\mathrm{CTRL}, n=6)$ and a high-fat diet group (HFD, $n=24$ ). There were no significant differences in body weights between the CTRL and HFD mice. For 20 weeks, the CTRL mice were fed with a standard chow diet and had ad libitum access to tap water; the HFD mice were fed with a high-fat diet (containing 10\% lard, 2\% cholesterol, $0.4 \%$ sodium cholate and $10 \%$ yolk powder) ad libitum in addition to the standard chow diet (the chow composition is listed in Supplementary Table S1). At the end of the 20th week, the HFD mice were divided into four subgroups: (1) HFD $(n=6)$; (2) EGCG (10 mg/kg; L-EGCG, $n=6)$; (3) EGCG (20 mg/kg; M-EGCG, $n=6$ ); and (4) EGCG (40 mg/ kg; H-EGCG, $n=6$ ). Mice in these four subgroups were fed with the high-fat diet for an additional 4 weeks. Mice in the EGCG groups received an intraperitoneal injection of EGCG $\left(10,20\right.$, and $40 \mathrm{mg} \cdot \mathrm{kg}^{-1} \cdot \mathrm{d}^{-1}$ body weight, respectively) for 4 weeks ${ }^{[15]}$. A $0.9 \% \mathrm{NaCl}$ solution was used as a sham injection to the non-EGCG groups. At the end of the 24th week, the food was removed and $12 \mathrm{~h}$ later, all the mice were anesthetized and euthanized. Blood was collected for biochemical analyses. Tissue specimens were excised, fixed in $4 \%$ paraformaldehyde or frozen immediately in liquid $\mathrm{N}_{2}$ and stored at $-80^{\circ} \mathrm{C}$ until analysis.

\section{Serum biochemical analysis}

Blood samples were allowed to clot, and the sera were isolated by centrifugation at 3000 rounds per minute for $8 \mathrm{~min}$ and then stored at $-20^{\circ} \mathrm{C}$ until assaying. The enzymatic activity of the glutamic-oxalacetic transaminase (GOT) and glutamic-pyruvic transaminase (GPT), and the serum lipid levels of triglycerides (TG), total cholesterol (TC), high density lipoprotein-cholesterol (HDL-C) and low density lipoproteincholesterol (LDL-C) were measured using commercial kits (Nanjing Jiancheng Bioengineering Institute, China) and a microplate reader (Bio-Rad 680, USA).

\section{Intraperitoneal glucose tolerance tests (ip-GTTs)}

In mice, ip-GTTs were carried out ( $2 \mathrm{~g}$ glucose $/ \mathrm{kg}$ body weight in $0.9 \% \mathrm{NaCl}$ ) after a $12 \mathrm{~h}$ fast. Blood samples were collected by tail bleeding at the indicated time points. Glucose was evaluated with glucose strips on a glucometer (ONETOUCH $^{\circledR}$ Ultra Easy, Johnson \& Johnson Ltd, USA). Serum insulin levels were assayed by enzyme linked immunosorbent assay (ELISA; Mercodia, Sweden). The area under the curve of blood glucose (AUCG), the area under the curve of serum insulin (AUCI), the HOMA insulin sensitivity index (HOMAISI) and the HOMA insulin resistance index (HOMA-IRI) were calculated as previously described ${ }^{[16-18]}$.

\section{Intraperitoneal insulin tolerance tests (ip-ITTs)}

In mice, ip-ITTs were carried out (0.5 IU insulin/ $\mathrm{kg}$ body weight in $0.9 \% \mathrm{NaCl}$ ) after a $4 \mathrm{~h}$ fast. Blood samples were collected by tail bleeding at the indicated time points. Glucose was measured with glucose strips on a glucometer (ONE$\mathrm{TOUCH}^{\circledR}$ Ultra Easy, Johnson \& Johnson Ltd, USA). The natural logarithms of the glucose measurements were taken, and the slope was calculated using linear regression (time $\times \mathrm{Ln}$ [glucose]) and multiplied by 100 to obtain the constant glucose decay rate during the insulin tolerance tests $\left(k_{\mathrm{ITT}}\right)$.

\section{Glucose-stimulated insulin secretion (GSIS) in pancreatic islet}

The islets were isolated by collagenase digestion, preincubated in Krebs-Henseleit buffer solution (KHBS) containing 0.5\% BSA and $2.8 \mathrm{mmol} / \mathrm{L}$ glucose, and equilibrated with $95 \% \mathrm{O}_{2}$ and $5 \% \mathrm{CO}_{2}$ at $37^{\circ} \mathrm{C}$ for $1 \mathrm{~h}$. Then, the medium was discarded, 
and the islets were incubated for an additional $1 \mathrm{~h}$ in $1 \mathrm{~mL}$ KHBS containing 2.8, 11.2, or $22.4 \mathrm{mmol} / \mathrm{L}$ glucose, respectively. Subsequently, the supernatant was collected to evaluate insulin secretion, the remaining islets were homogenized in KHBS, and the supernatant was collected for the determination of total insulin content by ELISA (Mercodia, Sweden).

\section{Hepatic histology and lipid content}

The liver and body weights were measured to calculate the liver indexes. To perform hematoxylin and eosin (H\&E) staining, a portion of liver tissues were fixed in $4 \%$ paraformaldehyde and embedded in paraffin. The frozen sections of the liver tissues were used to perform oil red $\mathrm{O}$ staining. To analyze the hepatic lipid content, additional portions of the liver tissues (50-100 mg) were collected. The TC and TG levels were measured according to the instructions of kits (Nanjing Jiancheng Bioengineering Institute, China), and the values were normalized to liver weights.

\section{In vivo insulin clearance test}

The serum insulin was evaluated during the ip-ITTs. The area under the curve of the insulin (AUCI) was calculated. The metabolic clearance rate of insulin (MCR-I) was evaluated as previously described ${ }^{[19]}$.

\section{Western blotting}

Proteins were extracted from mouse liver using RIPA lysis buffer with a protease inhibitor cocktail (Sigma-Aldrich, USA) and $1 \mathrm{mmol} / \mathrm{L}$ PMSF. The total protein levels of the lysates were determined using the Bradford method. Then, $50 \mu \mathrm{g}$ of protein were loaded and electrophoresed using SDS-PAGE, transferred onto polyvinylidene difluoride membranes (Amersham Biosciences, Bucks, UK), and incubated with rabbit monoclonal primary antibody to IDE (Abcam, USA) and then with peroxidase-conjugated secondary antibody. The immunocomplexes were visualized with enhanced chemiluminescence kits (Amersham Biosciences).

\section{Insulin-degrading enzyme activity assay}

The IDE activity was assessed by measuring the levels of protein extracts from the livers with the InnoZyme ${ }^{\mathrm{TM}}$ Insulysin/ IDE Immunocapture Activity Assay Kit (Calbiochem, Germany) and was expressed as relative fluorescent units.

\section{Statistical analysis}

The results are expressed as the mean \pm SD. Statistical analyses were performed using repeated measures ANOVA and oneway ANOVA. Differences were considered to be significant if $P<0.05$ or $P<0.01$. All of the calculations were performed using SPSS 13.0 software.

\section{Results}

EGCG improves metabolic disorders induced by a high-fat diet

Male C57BL/ 6 mice were fed a high-fat diet for 20 weeks to induce NAFLD. Then, the NAFLD mice were treated with low, moderate, or high doses of EGCG (ie, L-, M-, and H-EGCG, respectively) in the subsequent 4 weeks. The HFD mice had a significant enhancement in the body and visceral fat pad weights attributed to the increased energy intake, compared with the CTRL mice. In a dose-dependent manner, the EGCG treatment decreased energy intake and the body and visceral fat pad weights induced by the high-fat diet (Table 1).

Compared with the CTRL mice, the high-fat diet led to significant increases in fast blood glucose and insulin, as shown in Figure $1 \mathrm{~A}$ and $1 \mathrm{~B}$, and significant increases in serum lipid levels, including the TC, TG, and LDL-C levels (Figure 1C). The treatment of HFD mice with EGCG dose-dependently decreased the fasting blood glucose, serum insulin and lipid levels (TC, TG, and LDL-C). EGCG also dose-dependently increased HDL-C compared with HFD mice (Figure 1). These results suggest that the high-fat diet induces metabolic disorders, including hyperlipidemia, hyperinsulinemia and hyperglycemia, whereas EGCG dose-dependently improves the effects induced by a high-fat diet.

\section{EGCG improves insulin resistance induced by a high-fat diet}

There was a significant improvement in the fasting glucose and insulin levels in the EGCG groups. To verify these findings, we performed a homeostatic model assessment. The treatment of HFD-fed mice with EGCG improved insulin sensitivity and prevented insulin resistance induced by the high-fat diet, as evidenced by the HOMA-ISI and HOMA-IRI (Supplementary Figure S1).

Results from ip-GTTs showed the high-fat diet led to glucose intolerance (Figure 2A and 2B), accompanied by an insulin release peak delayed to 30 min after glucose loading, compared with an insulin release peak at 15 min after

Table 1. EGCG promotes weight loss in HFD mice. The values are expressed as mean \pm SD. $n=6$. ${ }^{\mathrm{c}} P<0.01$ vs $\mathrm{CTRL}$. ${ }^{\mathrm{e}} P<0.05$, ${ }^{\mathrm{f}} P<0.01$ vs HFD.

\begin{tabular}{|c|c|c|c|c|c|}
\hline & CTRL & HFD & L-EGCG & M-EGCG & H-EGCG \\
\hline Energy intake $\left(\mathrm{kcal} \cdot \mathrm{kg}^{-1} \cdot \mathrm{d}^{-1}\right)$ & $664.6 \pm 71.2$ & $922.1 \pm 104.7^{c}$ & $904.5 \pm 92.5^{c}$ & $790.4 \pm 91.5^{\text {ce }}$ & $676.2 \pm 80.2^{f}$ \\
\hline Carbohydrate intake $\left(g \cdot \mathrm{kg}^{-1} \cdot \mathrm{d}^{-1}\right)$ & $108.6 \pm 15.6$ & $98.9 \pm 11.3$ & $97.0 \pm 11.5$ & $84.6 \pm 9.7^{\mathrm{ce}}$ & $72.5 \pm 9.1^{\mathrm{cf}}$ \\
\hline Protein intake $\left(g \cdot \mathrm{kg}^{-1} \cdot \mathrm{d}^{-1}\right)$ & $36.2 \pm 5.7$ & $39.3 \pm 5.1$ & $38.5 \pm 5.1$ & $34.0 \pm 4.7$ & $28.8 \pm 4.3^{\mathrm{cf}}$ \\
\hline Fat intake $\left(g \cdot \mathrm{kg}^{-1} \cdot \mathrm{d}^{-1}\right)$ & $9.1 \pm 1.7$ & $40.7 \pm 4.7^{c}$ & $40.0 \pm 4.9^{c}$ & $34.9 \pm 4.3^{\mathrm{ce}}$ & $29.9 \pm 4.8^{\mathrm{cf}}$ \\
\hline Body weight (g) & $22.6 \pm 1.1$ & $29.2 \pm 1.6^{c}$ & $28.7 \pm 1.2^{c}$ & $25.5 \pm 1.0^{f}$ & $23.4 \pm 0.8^{f}$ \\
\hline Visceral fat pad (g) & $0.46 \pm 0.06$ & $1.35 \pm 0.12^{c}$ & $1.22 \pm 0.15^{c}$ & $0.77 \pm 0.07^{\mathrm{cf}}$ & $0.57 \pm 0.06^{f}$ \\
\hline
\end{tabular}

Food composition intake was calculated based on daily food intake and nominal calorie content of each individual item of the offered diet. At the end of the 24th week, all the mice were weighted, then anesthetized and euthanized. The visceral fat pad were excised and weighted. 
A
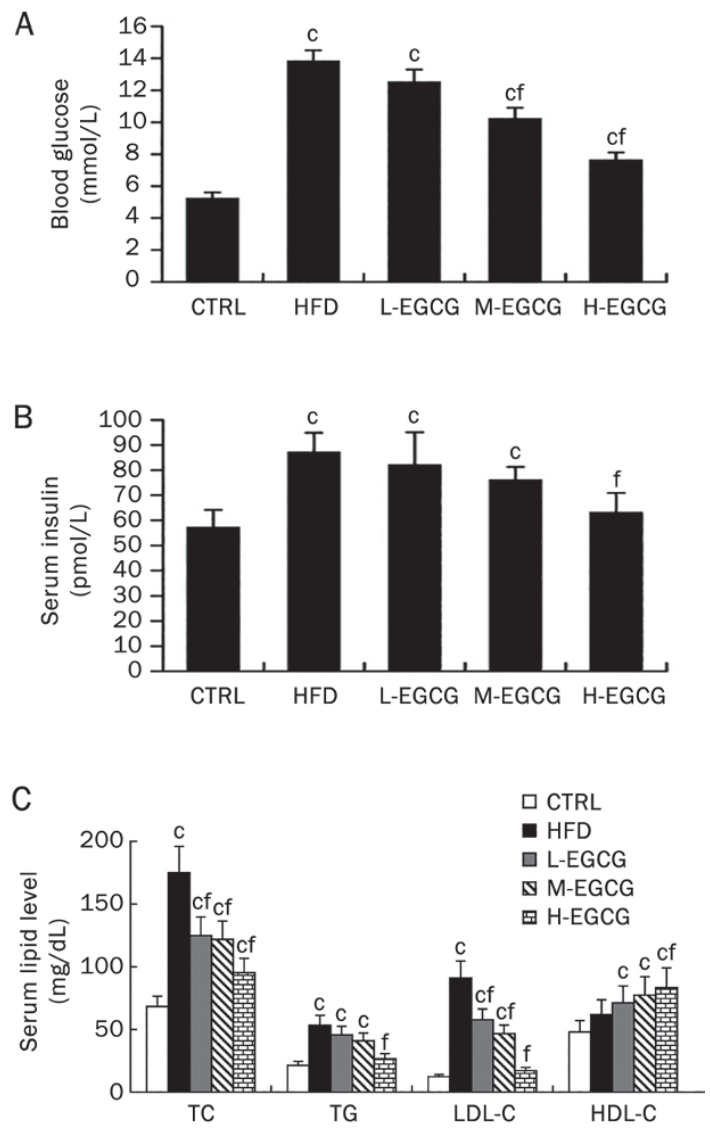

Figure 1. EGCG improves hyperlipidemia, hyperinsulinemia and hyperglycemia induced by a high-fat diet in mice. At the end of the 24th week, all the mice were anesthetized and euthanized. Blood was collected for biochemical analyses. The fasting blood glucose in mice (A), the fasting serum insulin in mice $(B)$, and the fasting serum lipids in mice (C). The values are expressed as the mean \pm SD. $n=6 .{ }^{c} P<0.01$ vs CTRL. ${ }^{f} P<0.01$ vs HFD.

glucose loading in the CTRL mice (Figure 2C). This suggested impaired insulin sensitivity in the HFD mice. EGCG dosedependently improved the glucose intolerance induced by the high-fat diet (Figure 2A and 2B). EGCG and especially H-EGCG altered the insulin dynamics and presented a higher insulin secretion peak at $15 \mathrm{~min}$, which was associated with a higher insulin concentration (Figure 2C and 2D).

During ip-ITTs, after intraperitoneal injection with insulin, the HFD mice showed a greater insulin tolerance with an increased AUCG and a reduced $k_{\text {ITT }}$ compared with the CTRL mice (Figure 3), which suggested that the high-fat diet induced insulin resistance. EGCG improved the insulin resistance induced by the high-fat diet in a dose-dependent manner, as confirmed by the increased glucose tolerance and insulin sensitivity (Figure 2) and the lower insulin tolerance compared with the HFD mice (Figure 3).

\section{EGCG rescues the insulin secretion in HFD mice}

To assess the insulin secretion levels, we performed GSIS assays on the pancreatic islets isolated from the mice. The high-fat diet led to an overall decreased GSIS level (Figure 4A) and decreased total insulin contents (Figure 4B). The insulin secretion in the HFD mice significantly decreased even after normalization to the total insulin content (Figure 4C). EGCG dose-dependently reversed the decreased insulin secretion and total insulin content induced by the high-fat diet (Figure 4).

\section{EGCG improves NAFLD induced by a high-fat diet}

We next evaluated the impact of EGCG on the hepatic morphology and the lipid accumulation in HFD mice. The hepatic histological analyses of the CTRL and HFD mice, according to the Brunt NAFLD score, revealed that all HFD mice had grade 2 or 3 fatty degeneration, which is mainly characterized by microvacuolar steatosis, approximately $67 \%$ of the HFD mice had lobular inflammation, and $67 \%$ and $33 \%$ of the HFD mice had grade 1 and grade 2 ballooning, respectively (Figure 5B and Table 2). No changes were found in the CTRL mice (Figure 5A and Table 2). Importantly, EGCG dose-dependently improved the steatosis, lobular inflammation and ballooning induced by the high-fat diet. Additionally, numerous hepatic cells regenerated in the EGCG-treated mice (Figure 5C-5E and Table 2). Oil red $\mathrm{O}$ staining in the frozen sections of the mouse liver tissue (Figure 5F-5J) demonstrated an abundant lipid accumulation in the hepatic cells of the HFD mice and that EGCG reduced the lipid accumulation induced by the high-fat diet in a dose-dependent manner (Figure 5F-5J).

We further quantitatively analyzed the effects of EGCG on the lipid contents in the liver and the hepatic function. As shown in Figure 5K, the high-fat diet led to an increase in the liver weight. The quantitative analysis of the lipids showed the TG and TC levels in the liver tissue of the HFD mice significantly increased compared with that of the CTRL mice (Figure 5L and 5M). The fasting GOT and GPT levels in the HFD mouse serum were clearly elevated (Figure $5 \mathrm{~N}$ and $5 \mathrm{O}$ ). EGCG dose-dependently decreased the liver weights and the contents of TG and TC in the liver (Figure 5K-5M). EGCG also dose-dependently reduced the GOT levels and especially the GPT levels (Figure 5N and 5O), which are considered markers of hepatic insulin resistance ${ }^{[20]}$. Taken together, these results demonstrated the effectiveness of EGCG for ameliorating NAFLD induced by a high-fat diet in mice.

\section{EGCG enhances insulin clearance and upregulates IDE in the liver of NAFLD mice}

Our results have shown that EGCG dose-dependently improved hyperinsulinemia and insulin resistance, and increased insulin sensitivity and secretion in NAFLD mice induced by a high-fat diet. EGCG induced variations in the decreased fasting serum insulin and the increased insulin secretion. We speculated that these variations could be due to the effects of EGCG on insulin clearance. We analyzed the effects of EGCG on insulin clearance in mice. During ip-ITTs, after the intraperitoneal injection of $0.5 \mathrm{IU}$ insulin/ $\mathrm{kg}$ body weight, the serum insulin levels at each time point in the HFD mice were significantly higher than those in the CTRL mice, as shown in the insulin curves (Figure 6A). The AUCI increased 
A
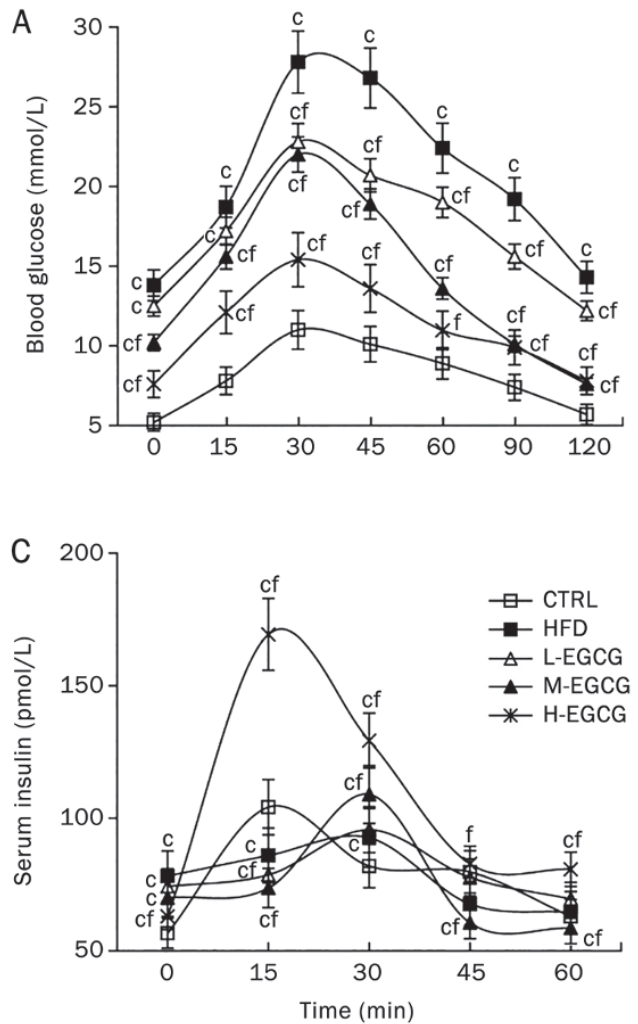

B

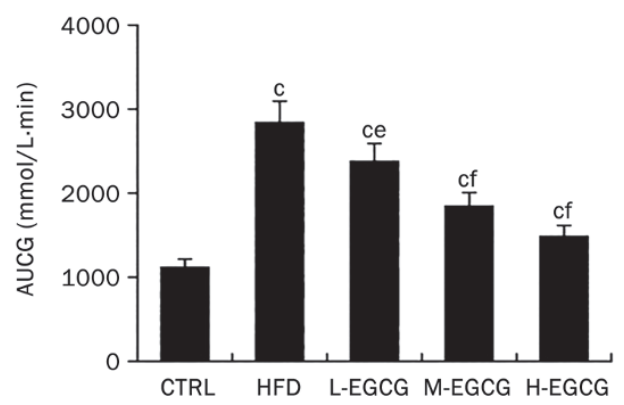

D

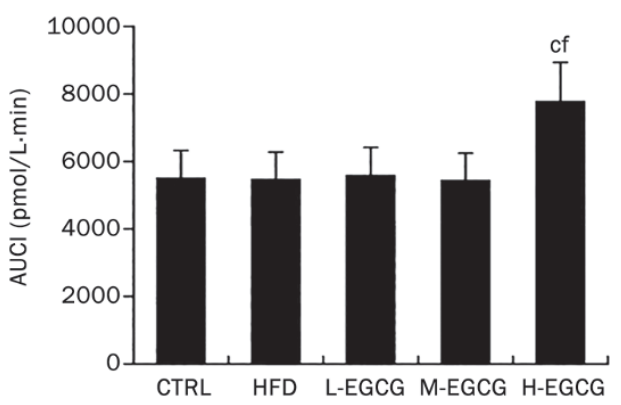

Figure 2. Effects of EGCG on glucose tolerance by ip-GTTs. At the 24th week, ip-GTTs were carried out after a $12 \mathrm{~h}$ fast. Blood samples were collected by tail bleeding at the indicated time points. Blood glucose and serum insulin were analyzed. The blood glucose curves (A), the AUCG (B), the insulin release curves (C), and the AUCI (D). The values are expressed as the mean \pm SD. $n=6$. $P$ values in (A) and (C) were calculated using repeated measures ANOVA. ${ }^{\mathrm{c}} P<0.01$ vs CTRL. ${ }^{\mathrm{e}} P<0.05,{ }^{\mathrm{f}} P<0.01$ vs HFD (by one-way ANOVA).

approximately 3-fold, whereas the MCR-I decreased approximately 3 -fold in the HFD mice compared with the CTRL mice (Figure 6B and 6C). This suggested that a high-fat diet leads to a diminished insulin clearance. Moreover, EGCG dosedependently reversed the diminishment in insulin clearance in NAFLD mice induced by the high-fat diet, as determined by the increased MCR-I (Figure 6C).

Approximately $50 \%-80 \%$ insulin is cleared by degradation by IDE in the liver ${ }^{[6]}$. Thus, we further analyzed the protein expression and activity of IDE in mouse liver. The IDE enzyme activity and protein levels in the liver were significantly decreased by the high-fat diet. EGCG increased the enzyme activity and protein expressions in the liver of NAFLD mice in a dose-dependent manner (Figures 6D and 6E).

\section{Discussion}

The many metabolic pathways of glucose and the lipid metabolism in the liver are controlled by insulin, which regulates the hepatic glucose output and lipid synthesis. Therefore, any transformation in hepatic insulin sensitivity is rapidly reflected in glucose homeostasis and TG level ${ }^{[21]}$. Insulin resistance has been considered an important pathophysiologic mechanism of obesity, dyslipidemia, diabetes, hypertension and NAFLD ${ }^{[1,2]}$. A series of studies have shown that insulin resistance is a key factor in the pathogenesis and potential evolution of hepatic steatosis. Insulin resistance is associated with increases in gluconeogenesis, peripheral lipolysis, the hepatic uptake of lipids and hepatocellular TG accumulation ${ }^{[22]}$. At present, there is a lack of effective and safe therapies for NAFLD. Although lifestyle intervention and weight loss is often advocated, it is difficult to maintain. Insulin sensitizers (eg, thiazolidinediones) improve steatosis and inflammation but induce significant weight gain. The use of antioxidants and other approaches have yielded conflicting and heterogeneous results. Thus, safer and more effective treatments for NAFLD should be explored.

Our study showed that the EGCG extracted from green tea dose-dependently improved steatosis, lobular inflammation and ballooning in the liver (Figure 5 and Table 2), and improved dyslipidemia (Figure 1) and hepatic function (Figure 5) in NAFLD mice induced by a high-fat diet. This suggests that EGCG is an effective treatment for NAFLD mice. Recently, several studies confirmed that the beneficial effects of EGCG on NAFLD may be attributed to its induction of hepatic autophagy and the attenuation of inflammation and oxidative stress ${ }^{[23-25]}$. Insulin resistance is closely associated with NAFLD and is a key factor in the pathogenesis and potential evolution of NAFLD ${ }^{[1,2]}$. However, the effect of EGCG on insulin resistance and its mechanism in treating NAFLD has rarely been discussed. In our study, EGCG allevi- 

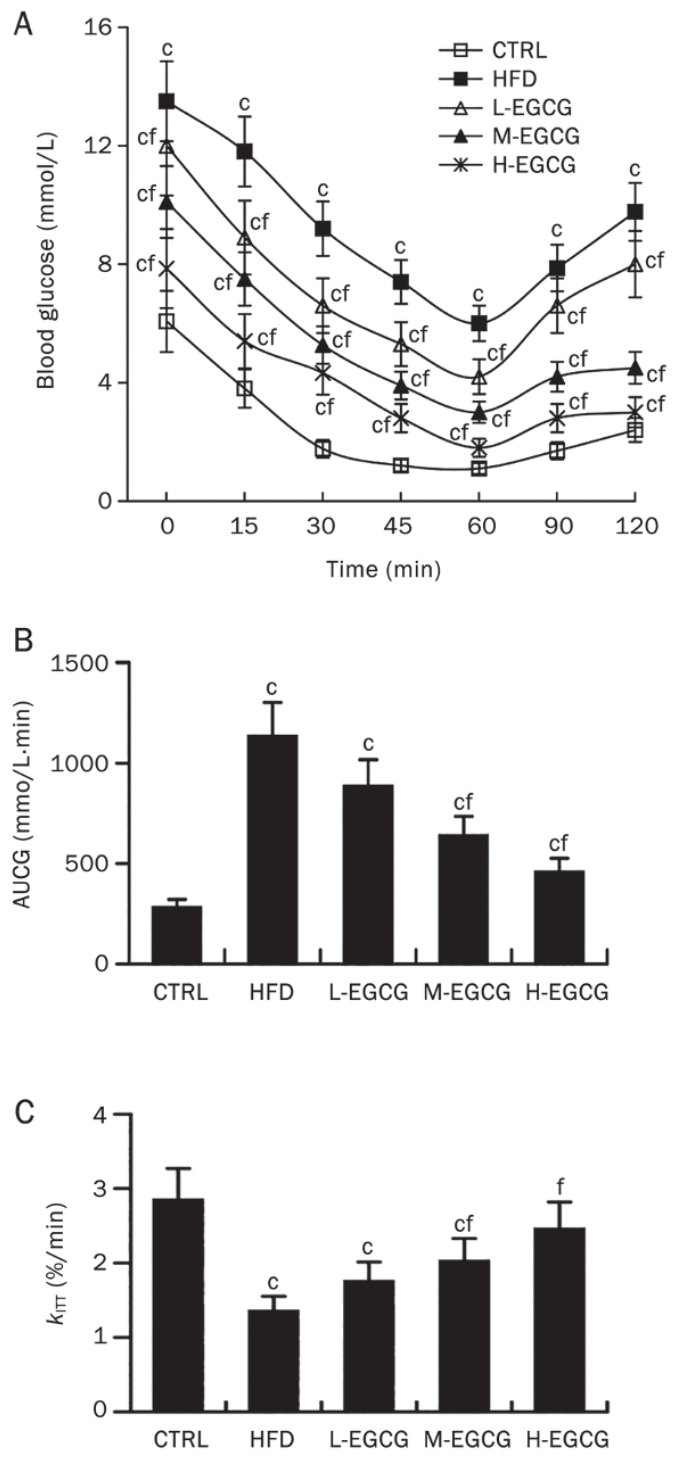

Figure 3. Effects of EGCG on insulin tolerance by ip-ITTs. At the 24th week, ip-ITTs were carried out after a $4 \mathrm{~h}$ fast. Blood samples were collected by tail bleeding at the indicated time points. Blood glucose was measured. The blood glucose curves (A), the AUCG (B), and the $k_{\text {Іाт }}(\mathrm{C})$. The values are expressed as the mean \pm SD. $n=6$. $P$ values in $(A)$ were calculated using repeated measures ANOVA. ${ }^{\mathrm{C}} P<0.01$ vs CTRL. ${ }^{\mathrm{f}} P<0.01$ vs HFD (by one-way ANOVA).

ated hyperinsulinemia (Figure 1), hyperglycemia (Figure 1) and insulin resistance (Figure 2 and 3 ) in a dose-dependent manner in NAFLD mice. This alleviation of insulin resistance by EGCG in NAFLD mice may be attributed to the weight loss effects of EGCG (Table 1), as previously shown wherein weight loss induced by diet control, exercise, pharmacotherapy and bariatric surgery improves insulin resistance ${ }^{[26,27]}$. However, more importantly, our study confirmed that an EGCG-induced increase in insulin clearance may contribute to the improvement of insulin resistance in NAFLD mice (Figure 6).

Several clinical studies have shown that impaired insulin
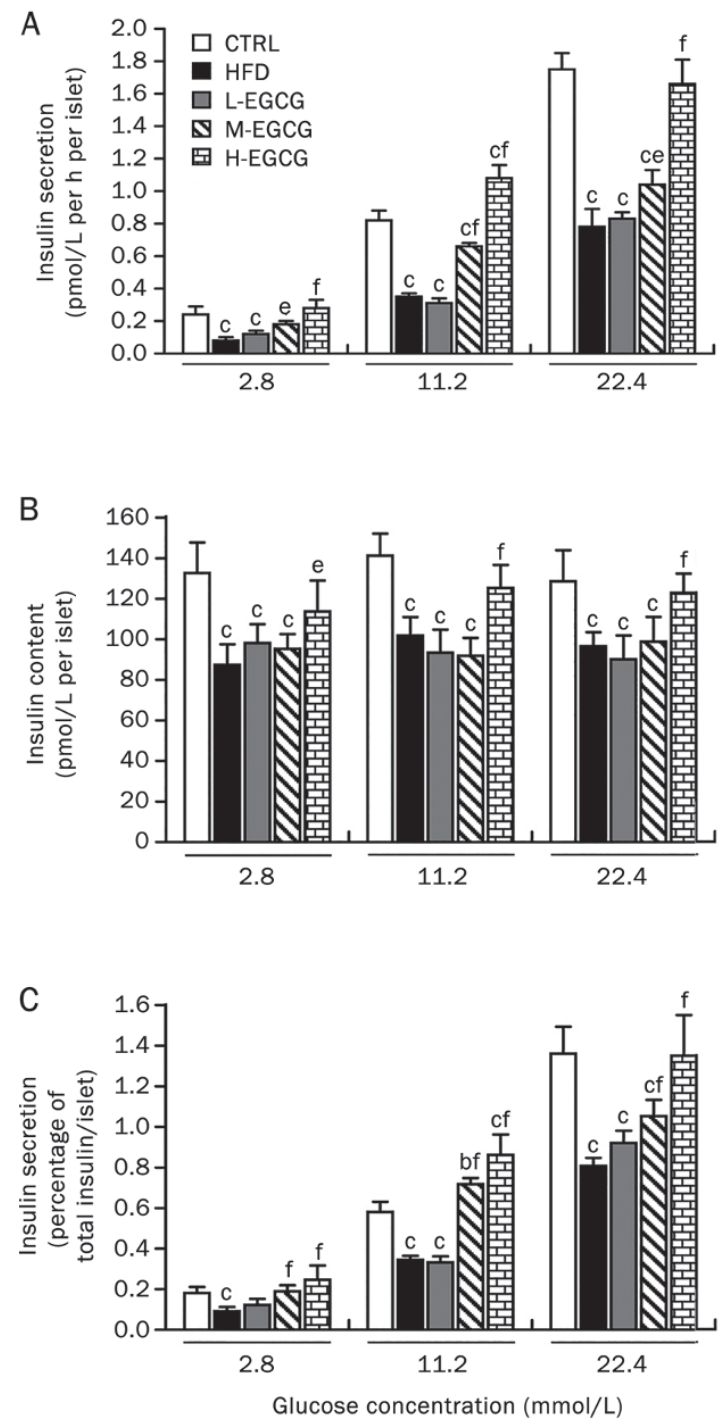

Figure 4. EGCG rescues the insulin secretion in HFD mice. At the end of the 24th week, all the mice were anesthetized and euthanized. The pancreatic islets were isolated by collagenase digestion, GSIS in pancreatic islet were performed: insulin secretion (A), total insulin content (B), and insulin secretion normalized by total insulin content (C). The values are expressed as the mean \pm SD. $n=6$. ${ }^{\mathrm{b}} P<0.05,{ }^{\mathrm{c}} P<0.01$ vs CTRL. ${ }^{\mathrm{e}} P<0.05,{ }^{\mathrm{f}} P<0.01$ vs HFD.

clearance, rather than insulin section, strongly correlated with hyperinsulinemia and insulin resistance in NAFLD patients ${ }^{[10,28]}$. Additionally, increased hepatic steatosis is associated with reduced insulin clearance and contributes to insulin resistance in non-diabetic Japanese individuals ${ }^{[29]}$. An experimental study demonstrated that primary hepatocytes treated with TG had a lower insulin clearance than control hepatocytes ${ }^{[30]}$. Together with our study results, this suggests that an impairment of insulin clearance may be a key factor that leads to insulin resistance in NAFLD. Additionally, this suggests that EGCG improves insulin resistance in NAFLD possibly through enhancing insulin clearance.

IDE has been recommended as a potential drug target in 

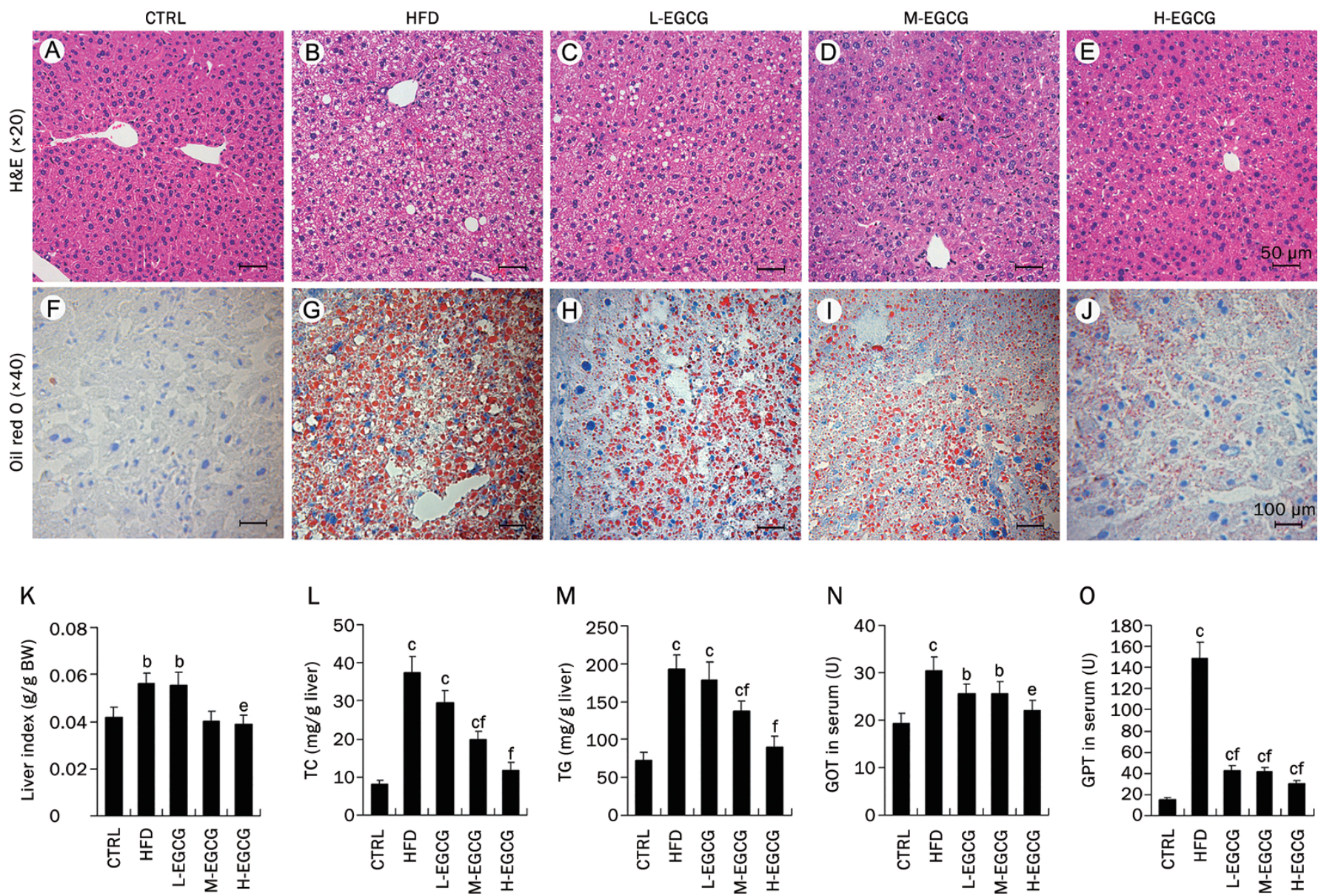

Figure 5. EGCG improves NAFLD induced by a high-fat diet. At the end of the 24th week, all the mice were anesthetized and euthanized. H\&E staining, oil red $\mathrm{O}$ staining and lipid quantitative analyses in mouse liver were performed. H\&E staining in the paraffin sections of hepatic tissues (A-E, magnification $\times 20$, scale bar $=50 \mu \mathrm{m})$, the oil red 0 staining in frozen sections of hepatic tissues ( $F-J$, magnification $\times 40$, scale bar $=100 \mu \mathrm{m})$, the liver indexes expressed as the liver weight (g) per g body weight (K), the TC content of liver (L), the TG content of liver (M), the GOT in serum (N) and the GPT in serum (0). The values are expressed as the mean \pm SD. $n=6$. ${ }^{b} P<0.05,{ }^{c} P<0.01$ vs $C T R L .{ }^{e} P<0.05,{ }^{f} P<0.01$ vs HFD.

Table 2. Grade of NAFLD in the liver of mice according to the Brunt NAFLD score.

\begin{tabular}{|c|c|c|c|c|c|c|c|c|c|c|c|}
\hline \multirow{2}{*}{$\begin{array}{l}\text { Groups } \\
(n=6)\end{array}$} & \multicolumn{4}{|c|}{$\begin{array}{l}\text { Steatosis (\%) } \\
\text { Grade }\end{array}$} & \multicolumn{4}{|c|}{$\begin{array}{l}\text { Lobular inflammation (\%) } \\
\text { Grade }\end{array}$} & \multicolumn{3}{|c|}{$\begin{array}{l}\text { Ballooning (\%) } \\
\text { Grade }\end{array}$} \\
\hline & 0 & 1 & 2 & 3 & 0 & 1 & 2 & 3 & 0 & 1 & 2 \\
\hline CTRL & 100 & 0 & 0 & 0 & 100 & 0 & 0 & 0 & 100 & 0 & 0 \\
\hline HFD & 0 & 0 & 33 & 67 & 33 & 67 & 0 & 0 & 0 & 67 & 33 \\
\hline L-EGCG & 0 & 33 & 67 & 0 & 50 & 50 & 0 & 0 & 50 & 50 & 0 \\
\hline M-EGCG & 0 & 50 & 50 & 0 & 83 & 17 & 0 & 0 & 100 & 0 & 0 \\
\hline H-EGCG & 0 & 83 & 17 & 0 & 100 & 0 & 0 & 0 & 100 & 0 & 0 \\
\hline
\end{tabular}

At the end of the 24th week, all the mice were anesthetized and euthanized. A portion of liver tissues in each mouse were fixed in $4 \%$ paraformaldehyde and embedded in paraffin. Then H\&E staining was performed. The hepatic lesions were evaluated according to the Brunt NAFLD score. There were six cases in each group. The values are expressed as incidence rates in mice.

the treatment of T2DM due to the IDE-catalyzed degradation of insulin ${ }^{[7]}$. The up-regulation of IDE activity may help to control hyperinsulinemia and insulin resistance in $\mathrm{T} 2 \mathrm{DM}^{[7-9,31]}$.
Unfortunately, not much is known about IDE regulation in NAFLD. We observed that EGCG dose-dependently induced IDE enzyme activity in the liver of NAFLD mice, which has 

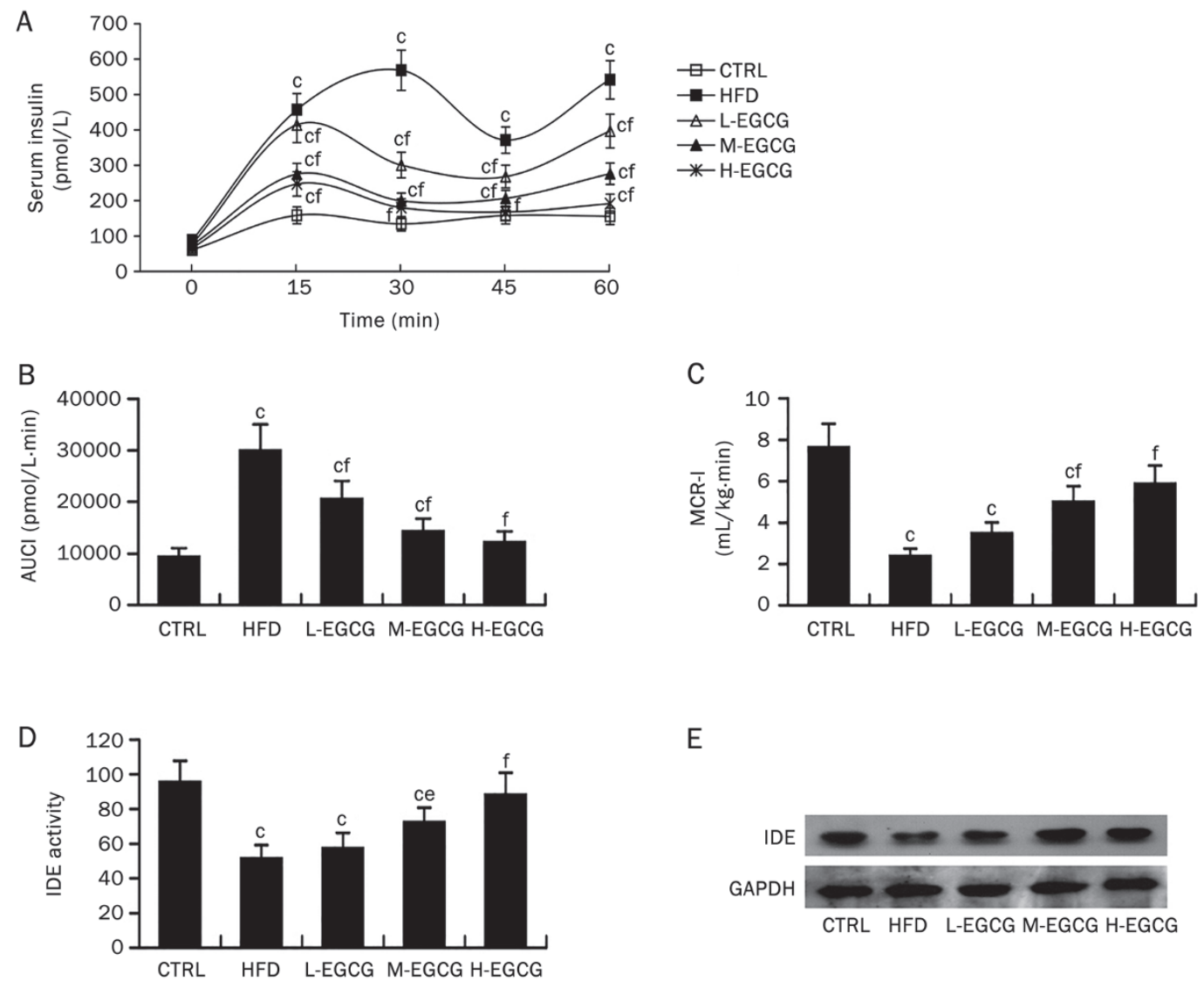

$\mathrm{E}$

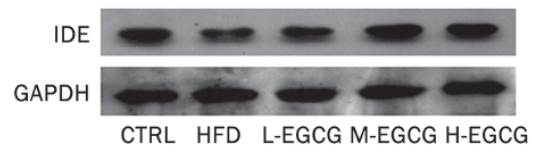

Figure 6. EGCG enhances insulin clearance and upregulates IDE in the liver of NAFLD mice. At the 24th week, insulin clearance was evaluated during the ip-ITTs. Then the mice were anesthetized and euthanized, the proteins were extracted from mouse liver to perform Western blotting. The insulin clearance test (A-C): the insulin curves (A), the AUCI (B) and the MCR-I (C). IDE activities in liver detected by immunocapture activity assay (D) and IDE expression in liver detected by Western blotting $(E)$. The values are expressed as the mean \pm SD. $n=6$. $P$ values in $(A)$ were calculated using repeated measures ANOVA. ${ }^{\mathrm{c}} P<0.01$ vs CTRL. ${ }^{\mathrm{e}} P<0.05,{ }^{\mathrm{f}} P<0.01$ vs HFD (by one-way ANOVA).

been associated with an increase in IDE protein expression; this represents a novel activity of EGCG in the pharmacological action. This activity suggested that EGCG may reduce insulin resistance by accelerating the clearance of insulin by IDE. However, this is a preliminary study, and the regulation mechanism of EGCG on IDE requires further investigation. The current knowledge about the IDE regulation mechanism is mostly derived from the study of the $\beta$-amyloid peptide in Alzheimer disease. In addition to insulin, IDE catalyzes the degradation of $\beta$-amyloid; IDE dysfunction is a critical factor in the pathogenesis of Alzheimer disease. In various studies on Alzheimer disease, researchers have discovered that the IDE gene promoter contains a peroxisome proliferatoractivated receptor- $\gamma$ (PPAR- $\gamma$ ) binding site, and PPAR- $\gamma$ induces IDE gene transcription in neuronal cells ${ }^{[32]}$. Furthermore, IDE gene transcription is induced by nuclear respiratory factor 1 (NRF1) ${ }^{[33]}$ and is reduced in neuronal cells by the Notch signaling proteins HES-1 and Hey- $1^{[34]}$. PPAR-ץ, NRF1, and Notch signaling will be further investigated to determine their roles in mediating the regulation of EGCG on IDE in NAFLD.

We demonstrated EGCG dose-dependently improved insu- lin resistance in NAFLD mice possibly not only by reducing body weight but also through enhancing the insulin clearance by hepatic IDE. The IDE protein expression and activity was induced by EGCG, suggesting a new action of EGCG in the treatment of NAFLD and the importance of IDE as a candidate for a targeted therapeutic approach for the treatment of NAFLD. Although this study was only conducted in the liver, these observations may apply to IDE regulation by EGCG in other tissues, such as kidney, brain and fat tissues.

\section{Acknowledgements}

This study was supported by the grants from National Natural Science Foundation of China (№ 31070687) and Guangdong Natural Science Foundation (№ 9451051501002535) and Science and Technology Planning Project of Guangdong Province, China (№ 2013B031800004).

\section{Author contribution}

Lu GAN designed research and acted as guarantor for the integrity of the entire study; Lu GAN, Zi-jun MENG, Ri-bo XIONG, and Jin-qiang GUO performed research; Fei ZOU, Bing-de LUO, and Hua LI contributed analytic tools; Xiao-cui 
LU, Zhi-wei ZHENG, and Yan-ping DENG analyzed data; Lu GAN wrote the paper.

\section{Supplementary information}

Supplementary information is available at website of Acta Pharmacologica Sinica.

\section{References}

1 Wang Y, Li YY, Nie YQ, Zhou YJ, Cao CY, Xu L. Association between metabolic syndrome and the development of non-alcoholic fatty liver disease. Exp Ther Med 2013; 6: 77-84.

2 Birkenfeld AL, Shulman GI. Nonalcoholic fatty liver disease, hepatic insulin resistance, and type 2 diabetes. Hepatology 2014; 59: 71323.

3 Marin-Juez R, Jong-Raadsen S, Yang S, Spaink HP. Hyperinsulinemia induces insulin resistance and immune suppression via Ptpn6/Shp1 in zebrafish. J Endocrinol 2014; 222: 229-41.

4 Kim B, McLean LL, Philip SS, Feldman EL. Hyperinsulinemia induces insulin resistance in dorsal root ganglion neurons. Endocrinology 2011; 152: 3638-47.

5 Lorenzo C, Hanley AJ, Wagenknecht LE, Rewers MJ, Stefanovski D, Goodarzi MO, et al. Relationship of insulin sensitivity, insulin secretion, and adiposity with insulin clearance in a multiethnic population: the insulin Resistance Atherosclerosis study. Diabetes Care 2013; 36: 101-3.

6 Fernandez-Gamba A, Leal MC, Morelli L, Castano EM. Insulindegrading enzyme: structure-function relationship and its possible roles in health and disease. Curr Pharm Des 2009; 15: 3644-55.

7 Haque R, Nazir A. Insulin-degrading enzyme: a link between Alzheimer's and type 2 diabetes mellitus. CNS Neurol Disord Drug Targets 2014; 13: 259-64.

8 Farris W, Mansourian S, Leissring MA, Eckman EA, Bertram L, Eckman $\mathrm{CB}$, et al. Partial loss-of-function mutations in insulin-degrading enzyme that induce diabetes also impair degradation of amyloid betaprotein. Am J Pathol 2004; 164: 1425-34.

9 Farris W, Mansourian S, Chang Y, Lindsley L, Eckman EA, Frosch MP, et al. Insulin-degrading enzyme regulates the levels of insulin, amyloid beta-protein, and the beta-amyloid precursor protein intracellular domain in vivo. Proc Natl Acad Sci U S A 2003; 100: 4162-7.

10 Bril F, Lomonaco R, Orsak B, Ortiz-Lopez C, Webb A, Tio F, et al. Relationship between disease severity, hyperinsulinemia, and impaired insulin clearance in patients with nonalcoholic steatohepatitis. Hepatology 2014; 59: 2178-87.

$11 \mathrm{Kim}$ HS, Quon MJ, Kim JA. New insights into the mechanisms of polyphenols beyond antioxidant properties; lessons from the green tea polyphenol, epigallocatechin 3-gallate. Redox Biol 2014; 2: 187 95.

12 Li Y, Zhao S, Zhang W, Zhao P, He B, Wu N, et al. Epigallocatechin3-O-gallate (EGCG) attenuates FFAs-induced peripheral insulin resistance through AMPK pathway and insulin signaling pathway in vivo. Diabetes Res Clin Pract 2011; 93: 205-14.

13 Jang HJ, Ridgeway SD, Kim JA. Effects of the green tea polyphenol epigallocatechin-3-gallate on high-fat diet-induced insulin resistance and endothelial dysfunction. Am J Physiol Endocrinol Metab 2013; 305: E1444-51.

14 Ueno T, Torimura T, Nakamura T, Sivakumar R, Nakayama H, Otabe S, et al. Epigallocatechin-3-gallate improves nonalcoholic steatohepatitis model mice expressing nuclear sterol regulatory element binding protein-1c in adipose tissue. Int J Mol Med 2009; 24: 17-22.

15 Ren Y, Deng F, Zhu H, Wan W, Ye J, Luo B. Effect of epigallocatechin3-gallate on iron overload in mice with alcoholic liver disease. Mol Biol Rep 2011; 38: 879-86.

16 Katz A, Nambi SS, Mather K, Baron AD, Follmann DA, Sullivan G, et al. Quantitative insulin sensitivity check index: a simple, accurate method for assessing insulin sensitivity in humans. J Clin Endocrinol Metab 2000; 85: 2402-10.

17 Del Prato S. Loss of early insulin secretion leads to postprandial hyperglycaemia. Diabetologia 2003; 46: M2-8.

18 Castell-Auvi A, Cedo L, Pallares V, Blay M, Ardevol A, Pinent M. The effects of a cafeteria diet on insulin production and clearance in rats. Br J Nutr 2012; 108: 1155-62.

19 Lee CC, Haffner SM, Wagenknecht LE, Lorenzo C, Norris JM, Bergman $\mathrm{RN}$, et al. Insulin clearance and the incidence of type 2 diabetes in Hispanics and African Americans: the IRAS Family Study. Diabetes Care 2013; 36: 901-7.

20 Vozarova B, Stefan N, Lindsay RS, Saremi A, Pratley RE, Bogardus C, et al. High alanine aminotransferase is associated with decreased hepatic insulin sensitivity and predicts the development of type 2 diabetes. Diabetes 2002; 51: 1889-95.

21 Wiernsperger N. Hepatic function and the cardiometabolic syndrome. Diabetes Metab Syndr Obes 2013; 6: 379-88.

22 Hassan K, Bhalla V, Ezz El Regal M, HH AK. Nonalcoholic fatty liver disease: A comprehensive review of a growing epidemic. World $J$ Gastroenterol 2014; 20: 12082-101.

23 Chen YK, Cheung C, Reuhl KR, Liu AB, Lee MJ, Lu YP, et al. Effects of green tea polyphenol (-)-epigallocatechin-3-gallate on newly developed high-fat/Western-style diet-induced obesity and metabolic syndrome in mice. J Agric Food Chem 2011; 59: 11862-71.

24 Kochi T, Shimizu M, Terakura D, Baba A, Ohno T, Kubota M, et al. Non-alcoholic steatohepatitis and preneoplastic lesions develop in the liver of obese and hypertensive rats: suppressing effects of EGCG on the development of liver lesions. Cancer Lett 2014; 342: 60-9.

25 Sae-Tan S, Grove KA, Kennett MJ, Lambert JD. (-)-Epigallocatechin3-gallate increases the expression of genes related to fat oxidation in the skeletal muscle of high fat-fed mice. Food Funct 2011; 2: 111-6.

26 Kievit P, Halem H, Marks DL, Dong JZ, Glavas MM, Sinnayah P, et al. Chronic treatment with a melanocortin-4 receptor agonist causes weight loss, reduces insulin resistance, and improves cardiovascular function in diet-induced obese rhesus macaques. Diabetes 2013; 62: 490-7.

27 Ballesteros-Pomar MD, Calleja S, Diez-Rodriguez R, Calleja-Fernandez A, Vidal-Casariego A, Nunez-Alonso A, et al. Inflammatory status is different in relationship to insulin resistance in severely obese people and changes after bariatric surgery or diet-induced weight loss. Exp Clin Endocrinol Diabetes 2014; 122: 592-6.

28 Kotronen A, Vehkavaara S, Seppala-Lindroos A, Bergholm R, YkiJarvinen $\mathrm{H}$. Effect of liver fat on insulin clearance. Am J Physiol Endocrinol Metab 2007; 293: E1709-15.

29 Goto T, Onuma T, Takebe K, Kral JG. The influence of fatty liver on insulin clearance and insulin resistance in non-diabetic Japanese subjects. Int J Obes Relat Metab Disord 1995; 19: 841-5.

30 Strang BD, Bertics SJ, Grummer RR, Armentano LE. Relationship of triglyceride accumulation to insulin clearance and hormonal responsiveness in bovine hepatocytes. J Dairy Sci 1998; 81: 740-7.

31 Abdul-Hay SO, Kang D, McBride M, Li L, Zhao J, Leissring MA. Deletion of insulin-degrading enzyme elicits antipodal, age-dependent effects on glucose and insulin tolerance. PLoS One 2011; 6: e20818.

32 Du J, Zhang L, Liu S, Zhang C, Huang X, Li J, et al. PPARgamma transcriptionally regulates the expression of insulin-degrading enzyme in primary neurons. Biochem Biophys Res Commun 2009; 383: 485-90.

33 Zhang L, Ding Q, Wang Z. Nuclear respiratory factor 1 mediates the transcription initiation of insulin-degrading enzyme in a TATA boxbinding protein-independent manner. PLoS One 2012; 7: e42035.

34 Leal MC, Surace El, Holgado MP, Ferrari CC, Tarelli R, Pitossi F, et al. Notch signaling proteins HES-1 and Hey-1 bind to insulin degrading enzyme (IDE) proximal promoter and repress its transcription and activity: implications for cellular $A \beta$ metabolism. Biochim Biophys Acta 2012; 1823: 227-35. 